\title{
A Coupled-Mode Filter Structure for Wireless Transceiver-Sensors using Reactive Loads
}

\author{
Thierry Laroche, Gilles Martin, William Daniau, \\ Sylvain Ballandras \\ FEMTO-ST, UMR 6174 CNRS-UFC-ENSMM-UTBM \\ Time\&Frequency Department, ENSMM \\ Besançon, France \\ sylvain.ballandras@femto-st.fr
}

\author{
Jean-Michel Friedt, Jean-François Leguen \\ SENSeOR SAS \\ TEMIS Innovation - Parc de Haute Technologie Lot\#3 \\ Besançon - Mougins, France \\ jmfriedt@femto-st.fr
}

\begin{abstract}
The possibility to remotely control impedance changes using resonant devices operating in the 434-MHz-centered Industry-Scientific-Medical (ISM) is demonstrated in this paper. The proposed principle is based on acoustically coupled modes in compact Surface Acoustic Wave (SAW) structures in which one port is connected to the variable load, the other being connected to a Radio-Frequency (RF) antenna for wireless interrogation purpose. The operation of the proposed principle is illustrated using a longitudinally-coupled resonator filter on quartz for "On-Off" switch remote control. Other sensor architecture are discussed as a conclusion of this work.
\end{abstract}

\section{INTRODUCTION}

Acoustic mode coupling using bulk or surface acoustic waves is widely used for the fabrication of bandpass filters on a spread radio-frequency spectrum $(5 \mathrm{MHz}-3 \mathrm{GHz})$ [1]. Its principle consists in coupling two identical resonators by a narrow area where evanescent fields generated by each device can develop and overlap, creating the mode coupling conditions. Such a structure allows for transmitting information using this coupling effect between input and output ports of the structure in a spectral band corresponding to the coupling efficiency of the two resonators and the coupling strength of the modes.

The idea proposed in this work consists in exploiting this coupling property for the wireless measurement of given physical parameter acting on a variable reactive load connected to one port of the structure, the other being connected to an antenna. This approach is developed for resonant sensors operating at $434 \mathrm{MHz}$ (in the corresponding ISM band) in a very similar way to reflective $2.45 \mathrm{GHz}-$ centered delay lines with reflection modulated by variable reactive load as well [2]. For resonators operating in the 434 $\mathrm{MHz}$ ISM band, measuring electrical characteristics influenced by parametric changes (capacitance, selfinductance, resistance) reveals challenging using standard single port resonators. Particularly, a reliable and accurate measurement requires differential sensor structures [3]. This approach generally is achieved using two resonators with one loaded by the variable charge and the other is used as reference. In the proposed approach, only one resonator structure is used, yielding compact sensor configurations, with two modes naturally exhibiting the same (or almost the same) parametric sensitivity but a differential sensitivity to the variable load changes.

The paper presents theoretical simulation results considering Longitudinally-Coupled Resonator (LCR) structures as a representative configuration for illustrating the interest of the proposed approach which can be generalized to any kind of coupled mode waveguides and resonators (FBAR, HBAR, etc.). An experimental implementation is reported on quartz at $434 \mathrm{MHz}$, using resistance load changes as an example in an "On-Off" remote control approach. Frequency changes of about $70 \mathrm{kHz}$ are measured, which is found to be largely above the residual thermal sensitivity of the two-mode temperature sensitivity and conforms theoretical predictions. Wireless interrogation of such a device is finally assessed, although the $\mathrm{Q}$ factor of each resonance was not optimized in the proposed work.

The first section of the paper describes the general principle of the proposed concept. Theoretical analysis of the sensitivity to capacitance and resistance changes of a LCR on $(\mathrm{AT}, \mathrm{X}) \mathrm{Quartz}$ is reported, followed by the experimental work description and result report. As a conclusion, the possibility to transpose the approach to other coupled-mode resonant structures is discussed.

\section{Basic Principle of Acoustic Mode Coupling}

\section{A. Remote control of variable impedance loads}

As explained in introduction, reflective delay lines built on $\mathrm{LiNbO}_{3}$ are particularly well suited to remotely control changes of impedance loads connected to one group of reflector [2]. Due to the high electromechanical coupling of Rayleigh wave on such substrates, changing the electrical boundary conditions of the above reflector group yields 
substantial changes in the device electrical signature. This feature offers an efficient way to remotely monitor the parameters at the origin of the said impedance changes, particularly for classical impedance loads (resistor, inductors, capacitors) which do not basically benefit from specific RF transmission capabilities. The SAW device then is mainly used as a transponder and can be optimized in that way, regardless specific sensitivity optimization which may require abusive efforts compared to the actual corresponding benefit. However, for specific applications, reflective delay lines may not reveal adapted for various reasons (compactness, environment robustness, etc.) and therefore the possibility to implement alternative devices (such as resonators operating in the 434-MHz-centered ISM band) is expected to complete the art and the engineers solution palette. SAW resonators can actually be frequency-pulled by inserting variable capacitors or inductors in parallel or in series with the resonator and the antenna, yielding immediate demonstration of the corresponding functionality assuming that the charge values are matched with those of the resonator (static capacitance and motional parameters as well). This approach however is limited by the existence of other frequency-pulling effects related to the RF link changes for instance, which therefore cannot be identified nor separated from the useful information which is looked for. To address this problem, the use of an additional reference resonator is the usual way to proceed. However, this approach generates other difficulties related to the effective control of the resonator characteristics (resonance frequency, thermal sensitivity, etc.) which pushes to investigate more robust alternative solutions. In that purpose, the reflective delay line structure has been analyzed and used as an inspiration to try and address the above mentioned difficulties. Particularly, one major motivation was to identify resonant structures potentially exhibiting different modes with different parametric sensitivities. The use of dispersive waveguide (based on layered structures for instance) which may advantageously answer this feature however was prohibited because of the additional difficulty to control the selected working point. Moreover, structures exhibiting at least two modes close enough in the spectral domain to simultaneously operate in the ISM band using a single transducer electrode configuration is assumed beyond easy reach. Another advantageous aspect of the reflective delay line is related to the use of only one transducer for RF interrogation purpose. Beyond the device simplicity, the use of a single transducer connected to the RF antenna (instead of two resonators in parallel as usually achieved with SAW resonators in the 434-MHz ISM band) is expected to improve the sensor robustness versus RF link variations. Therefore, the investigated resonant device must allow for connecting the variable impedance load at one edge and the RF antenna on the other edge, limiting as much as possible the impact of the later on the overall device response. To answer all the requirements at once, coupled-mode resonators were identified as one of the adapted solution and therefore has been analyzed more in-deep in the next section.

\section{B. Basic Principle of Acoustic Mode Coupling}

acoustic mode coupling is generally achieved between at least two identical transducers separated by a gap in which the acoustic waves exhibit an evanescent behavior. The corresponding penetration depth must be long enough to allow for spacial overlapping of the fields, yielding the possibility for the said transducers to exchange energy one another. Such an energy exchange can happen only if the excitation boundary conditions for both transducers are satisfied simultaneously. It has been shown by many authors [4] that two configurations fulfill this requirement, i.e. a symmetrical or an anti-symmetrical distribution of acousto-electric energy within the transducers. The scheme of fig. 1 illustrates the mode distribution within a SAW LCR filter.

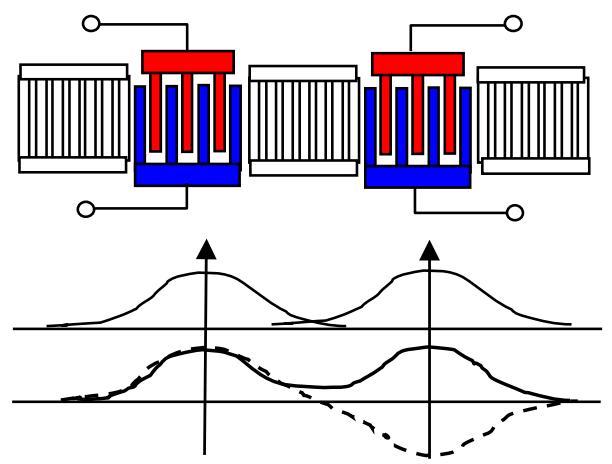

Figure 1.Typical structure and mode distribution of a LCR filter - two transducers are coupled via a coupling cavity designed to force the evanescent regime of the wave at the transducer synchronicity frequency

The behavior of such device can be efficiently represented using Coupling-of-Mode $(\mathrm{CoM})$ or mixed-matrix approaches. For filter design, the most important parameter to optimize is of course the transfer function $\mathrm{S}_{21}$ as it predominantly governs the effective operation of the device. In this work, the device is used as a single-port RF device although its electrical response exhibits two distinct modes as shown in the theoretical response of a LCR filter on (AT,X) Quartz (fig.2). the design parameters of this configuration (Mirror 400 strips and a period $=3.58 \mu \mathrm{m}$, IDT 50 fingers and a period $=3.56 \mu \mathrm{m}$, coupler 25 strips and a period $=3.58 \mu \mathrm{m}, 250 \mathrm{~nm}$ thick $\mathrm{Al}$ electrodes) have been used in section III for all the simulations. The aperture was the only varied parameter for adjusting the pulling capability of the device.

Practically, there is no reason to associate one mode and one transducer together as both transducer interacts one another to compose the corresponding spectral behavior. The latter actually is conditioned by key parameters either related to the fundamental properties of the SAW on the used substrate or to the electrode grating and transducer combination. Particularly for the LCR filter, the length of the coupling cavity as well as the magnitude of the associated reflection coefficient directly impacts the frequency distance between the two modes. For a mode resonating at the 
beginning of the frequency stop-band, the stop-band of the Inter-Digitated Transducer (IDT) must be slightly smaller than the one of the coupling and mirror grating in that purpose. On the other hand, the frequency-pulling efficiency is mainly governed by the intrinsic Rayleigh wave characteristics, as well as thermal and other parametric sensitivities. The number of electrodes in the IDT as well as the acoustic aperture also play a notable role for matching the device

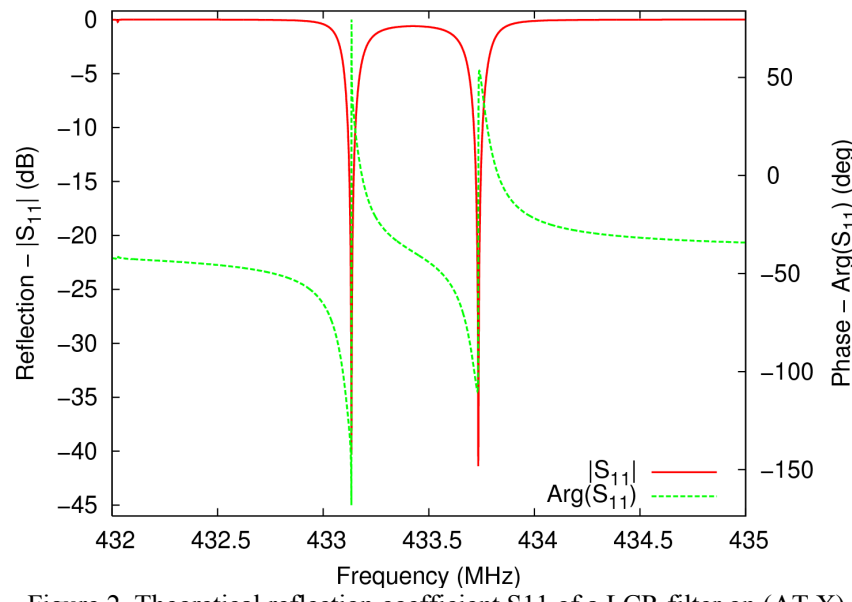

Figure 2. Theoretical reflection coefficient S11 of a LCR filter on (AT,X) quartz designed to operate in the 4324-MHz ISM band

\section{LCR-Sensor Theoretical Sensitivity to Load Changes}

In this section, a brief and simple analysis of the frequency-pulling capability of a LCR sensor on (AT,X) Quartz is reported. As explained above, the SAW device is characterized using a mixed-matrix approach, providing the admittance matrix of the corresponding quadrupole

$$
\left\{\begin{array}{l}
i_{1} \\
i_{2}
\end{array}\right\}=\left[\begin{array}{ll}
Y_{11} & Y_{12} \\
Y_{21} & Y_{22}
\end{array}\right]\left\{\begin{array}{l}
V_{1} \\
V_{2}
\end{array}\right\}
$$

The simulation of the pulling capabilities of the device then is simply achieved by applying electrical boundary conditions to the above admittance matrix (fig.3).

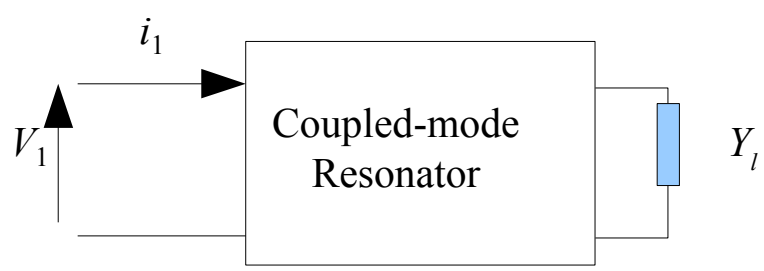

Figure 3. Block-diagram of the LCR sensor - one port is loaded by a variable impedance and the sensitivity of the device is provide by the port\#1 electrical response

The application of the load $Y_{l}$ on the port\#2 of the quadrupole allows to derive the resulting admittance at port\#1 $Y_{11}$ as follows

$$
i_{2}=Y_{l} V_{2} \text { yields } Y_{11}^{\prime}=Y_{11}+\frac{Y_{12} Y_{21}}{Y_{l}-Y_{22}}
$$

The admittance load is then replaced either by a constant value to simulate purely resistive charges or by frequency varying functions representing either a capacitance or a selfinductance. Figure 4 shows the impact of a variable resistance load on a LCR sensor on Quartz simulated using the abovedescribed scheme. In this specific case, the induced frequency variation ranges correspond to a device with a $140 \lambda$ aperture. From 10 to $1 \mathrm{k} \Omega$, both resonance are notably dumped and an almost linear frequency change of 60 and $80 \mathrm{~Hz}$ per $\mathrm{Ohm}$ is predicted for the first and second resonance respectively. The total differential frequency variation between the two modes form almost short to almost open circuit is $40 \mathrm{kHz}$. Although modest, these values are large enough to be measured using wired and wireless electronics as well. Increasing the aperture (fig.5) allows for tuning the sensitivity range of the sensor (now from about 100 to $10 \mathrm{k} \Omega$ ).

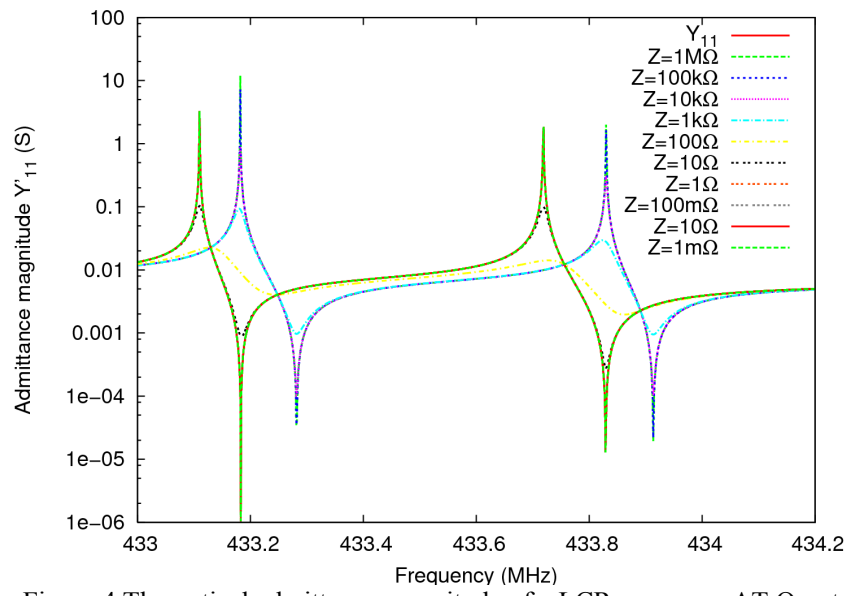

Figure 4.Theoretical admittance magnitude of a LCR sensor on AT Quartz for a pure resistance load varying from $1 \mathrm{~m}$ (short) to $1 \mathrm{M} \Omega$ (open), $\mathrm{W}=140 \lambda$

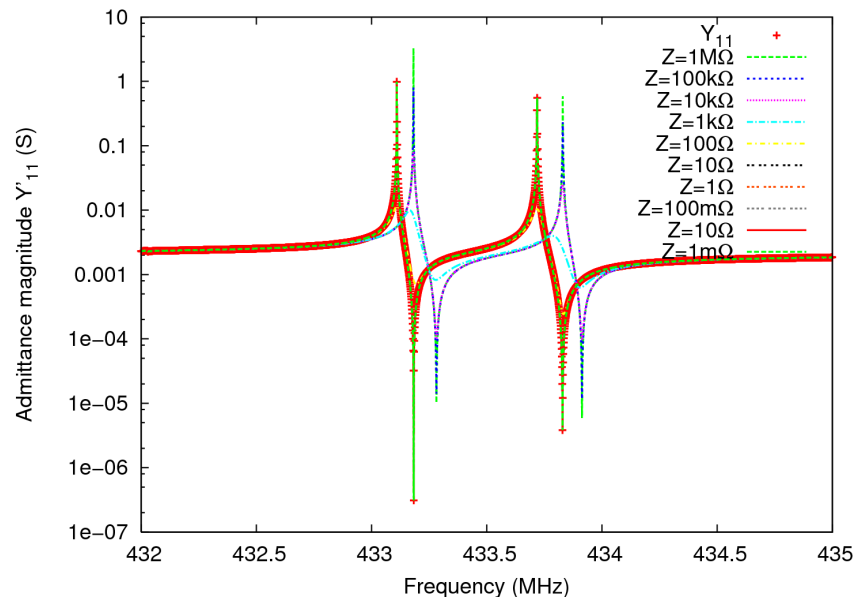

Figure 5: Theoretical admittance magnitude of a LCR sensor on AT Quartz for a pure resistance load varying from $1 \mathrm{~m}$ (short) to $1 \mathrm{M} \Omega$ (open), $\mathrm{W}=40 \lambda$ 
The same situation almost occurs for pure capacitive loads, as shown in figs. 6 and 7. The same coupled structure as the one used to determine the sensitivity to resistance load has been considered here. One can note that in this configuration, no magnitude drop is observed on the harmonic admittance is the capacitance is assumed perfect (no associated losses) but the second resonance is more affected than the first one when changing the capacitive load, yielding a total differential sensitivity of more than $40 \mathrm{kHz}$ on the full range. The device is particularly sensitive to capacitance variations in the 0.1 $10 \mathrm{pF}$ range as its aperture corresponds to $140 \lambda$ as previously considered for the sensitivity to resistance loads.

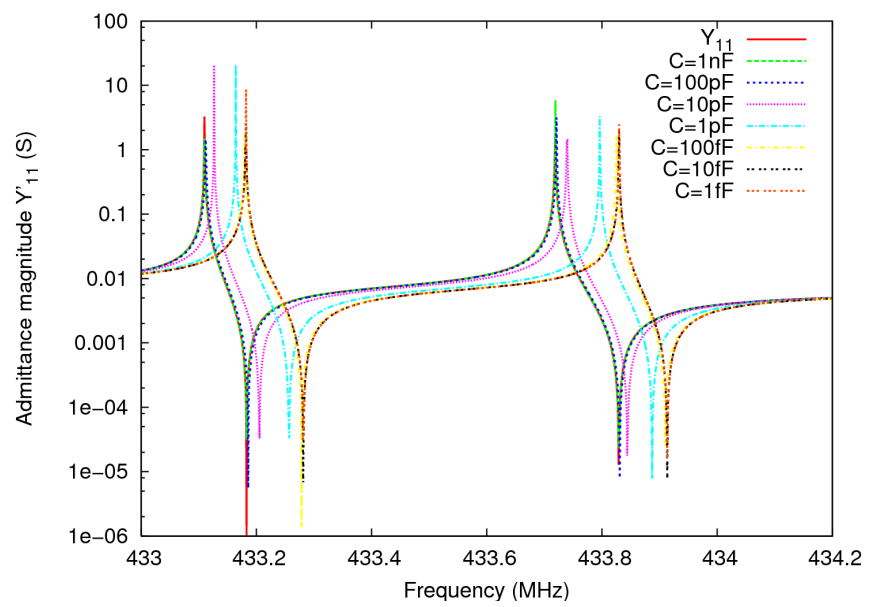

Figure 6: Theoretical admittance magnitudes of a LCR sensor on AT Quartz for a pure capacitance load varying from $1 \mathrm{f} F$ to $1 \mathrm{nF}, \mathrm{W}=140 \lambda$

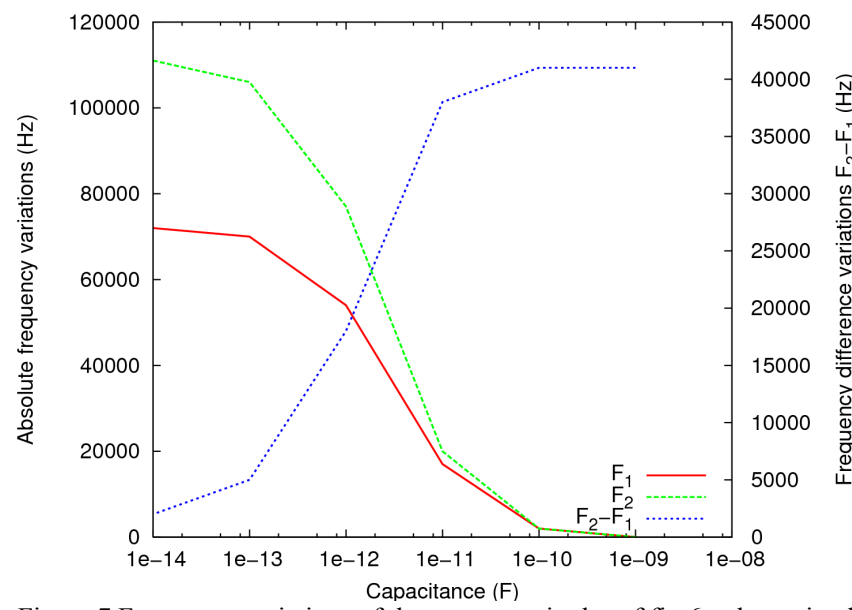

Figure 7.Frequency variations of the max magnitudes of fig. 6 - the optimal sensitivity zone is between 0.1 and $10 \mathrm{pF}$, the static capacitance of the SAW LCR sensor is close to $3 \mathrm{pF}$

Here again, the aperture can be advantageously used to optimize the capacitance range for which the device will exhibit the largest sensitivity (figs. and obtained for a 40 aperture). Note also that for increasing this range, more electromechanical (than the one of Rayleigh wave on AT quartz) coupling is mandatory.

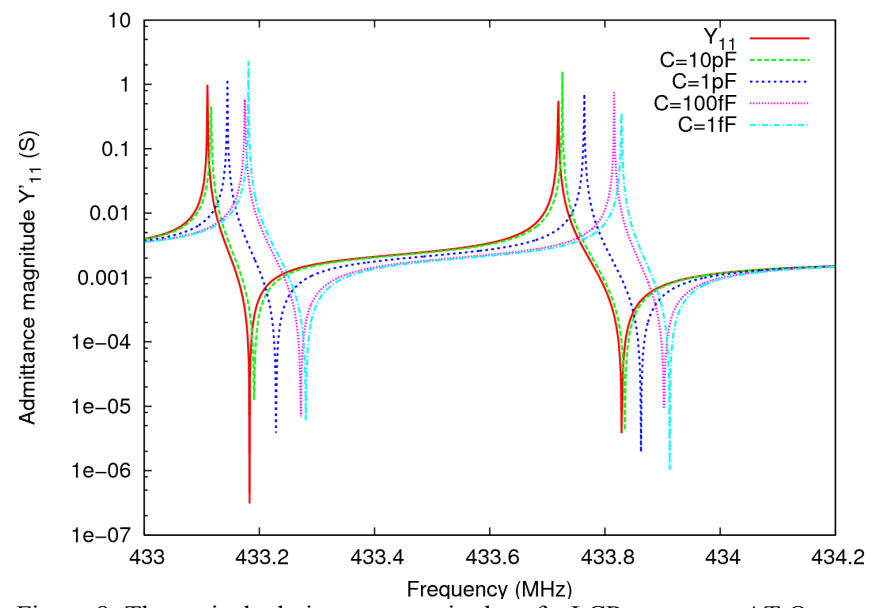

Figure 8: Theoretical admittance magnitudes of a LCR sensor on AT Quartz for a pure capacitance load varying from $1 \mathrm{~F}$ to $1 \mathrm{nF}, \mathrm{W}=40 \lambda$

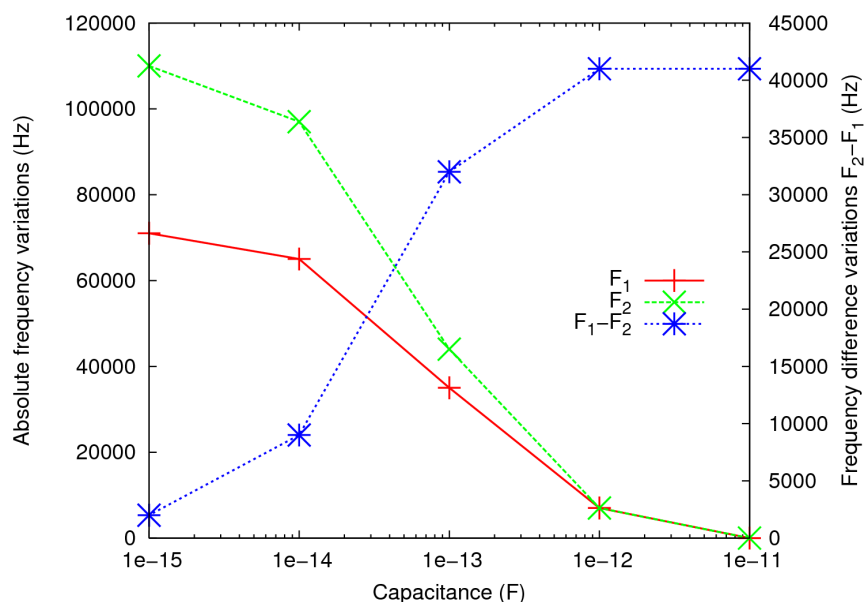

Figure 9: Frequency variations of the max magnitude of fig. 8 - the optimal sensitivity zone is between $10 \mathrm{fF}$ and $1 \mathrm{pF}$, the static capacitance of the SAW LCR sensor is close to $3 \mathrm{pF}$

\section{Experimental Assessment}

To test the actual operation of such a device for remotely monitoring load variations, a SAW LCRF has been built on (AT,X) Quartz along the above reported technology parameters used to compute the theoretical device sensitivity (section III). Figure 10 shows the experimental reflection coefficient of the LCR sensor measured with a network analyzer when submitted to open and load circuit conditions on the sensing side. For both resonances, a frequency shifted of about $60 \mathrm{kHz}$ has been observed, which agrees rather well with the theoretical predictions. However, the corresponding differential sensitivity reveal very small (about $10 \mathrm{kHz}$ ) contrarily to the expected $40 \mathrm{kHz}$. This result is discussed further when analyzing wireless measurements. 


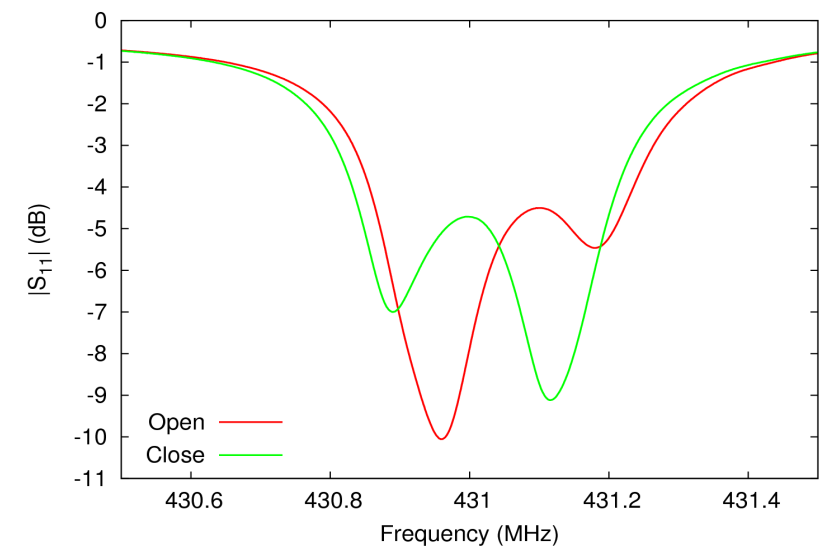

Figure 10: Experimentally measured $S_{11}$ parameter of a LCR sensor built on Quartz submitted to short and open conditions at its sensing port

Whatever this unexpected result, an experimental set-up has been developed to assess the possibility for remotely interrogating such a coupled-mode resonator sensor associated with a "On-Off" switch. Figure 11 shows the implemented system composed a printed circuit on which the packaged SAW device $\left(7 \times 9 \mathrm{~cm}^{2}\right.$ ceramic package) is bonded together with the loading switch. The switch is toggled manually and the device response is recorded using a RF reader commonly developed by SENSeOR and FEMTO-ST [5]. Figure 12 shows the resulting measurements under the form of absolute frequency variations whereas fig. 13 Reports the deduced differential frequency variations, simply subtracting both mode resonance frequencies of the coupled resonator sensor. Although it is clear that the absolute frequency variations are larger than the resulting relative frequency difference, the interest of the later information is its robustness to parametric perturbation. In that purpose, the temperature coefficient of frequency (TCF) of both modes and the resulting temperature sensitivity of the frequency difference have been measured.

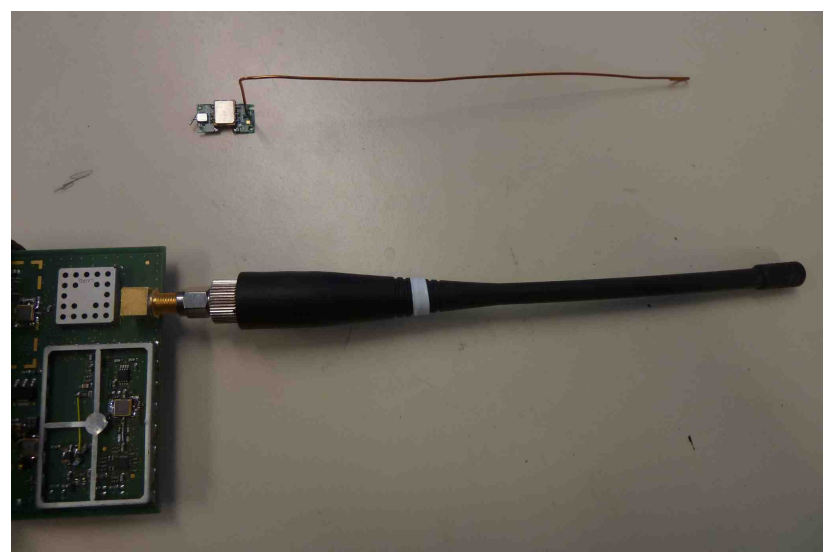

Figure 11.Photograph of the set-up used to assess the "On-Off" remote control using a simple switch connected at the probing side of the LCR sensor

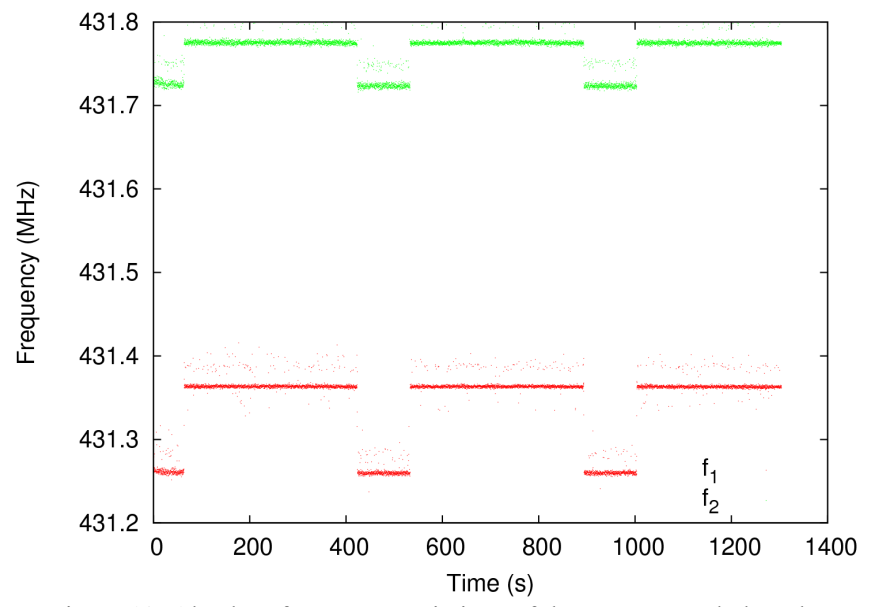

Figure 12: Absolute frequency variations of the sensor coupled modes generated by the switch toggling

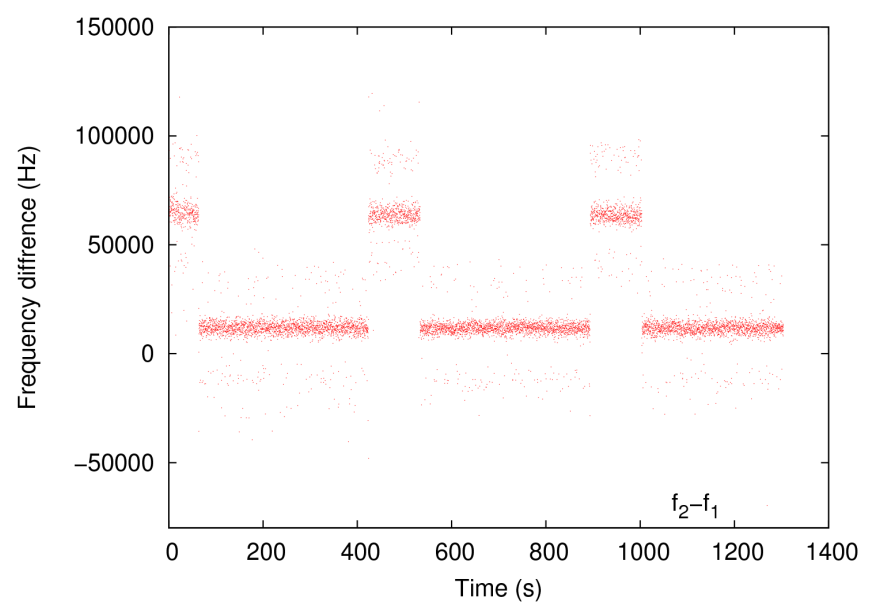

Figure 13: Differential frequency variations obtained by subtracting both frequencies in fig12

The results of the temperature-frequency measurements are reported in figs.14 and 15. The best fit of the evolution of both modes vs temperature yields $\mathrm{TCF}_{1}$ equal to $-3.93 \mathrm{ppm} . \mathrm{K}^{-1}$ for the mode\#1 and $-3.85 \mathrm{ppm} . \mathrm{K}^{-1}$ for the mode\#2 (corresponding $\mathrm{TCF}_{2}$ are equal to -38.5 and -44.7 ppb.K ${ }^{-2}$ ). In both cases, the absolute frequency variations on the whole temperature range $\left(0-100^{\circ} \mathrm{C}\right)$ always exceed $200 \mathrm{kHz}$, i.e. much larger than the frequency variation due to the switch toggling (fig. 12, 110 and $50 \mathrm{kHz}$ for the mode \#1 and \#2 respectively). The characterization of the TCF of the frequency difference yields $\mathrm{TCF}_{1}$ and $\mathrm{TCF}_{2}$ respectively equal to $80 \mathrm{ppm} . \mathrm{K}^{-1}$ and $6.4 \mathrm{ppm} . \mathrm{K}^{-2}$ for a reference frequency of $412 \mathrm{kHz}$ and a turnover temperature close to $30^{\circ} \mathrm{C}$. Although these figures look quite large, they indicate a total frequency variation of about $10 \mathrm{kHz}$ on the whole temperature range, i.e. much smaller than the frequency variations induced by the switch toggling $(\sim 65 \mathrm{kHz})$. Consequently, the use of the frequency difference allows for a reliable and robust switch 
monitoring on the whole temperature excursion, contrarily to the use of absolute frequencies of the device modes.

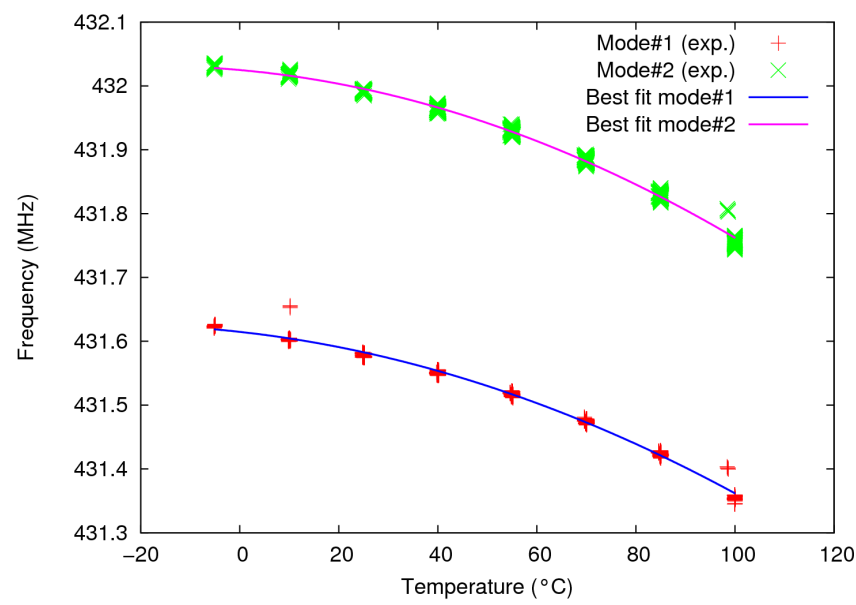

Figure 14: Temperature coefficient of frequency (TCF) of the two modes of the AT-cut Quartz LCR sensor

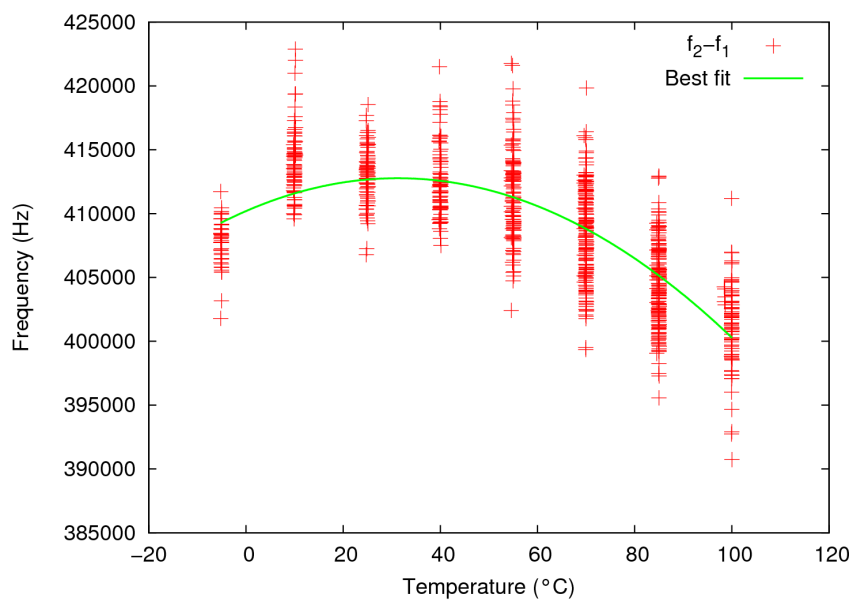

Figure 15: Temperature coefficient of the frequency difference of the two modes of fig. 14

\section{CONCLUSION}

In this paper, theoretical simulation results and first experimental assessment using a longitudinally-coupled resonator filter architecture as a transponder for remotely monitoring reactive load changes. The interest of such device is that it allows for the wireless interrogation of resonant devices with a natural differential regime expected to exhibit a high robustness to parametric effects (temperature, stress, etc.). Computations have been achieved considering a LCR transponder built on AT-Quartz operating in the 434-centered ISM band. The whole differential frequency variation of the device due to pure resistive and capacitive load changes has been found theoretically equal to $45 \mathrm{kHz}$ in the corresponding ranges. The excursion of the frequency difference is mainly conditioned to the wave characteristics (electromechanical coupling) whereas the absolute capacitance and resistance values for which the device exhibits the largest drift is related to the device features (mode coupling, aperture, etc.).

An experimental implementation has been achieved using a quartz LCRF operating near $434 \mathrm{MHz}$ in an "On-Off" remote control approach. Wireless interrogation of such a device has been assessed and the robustness of the approach vs temperature has been demonstrated, yielding an effective remote control of a bi-static switch on a 0 -to- $100^{\circ} \mathrm{C}$ temperature range. Further work will be devoted to increasing the sensitivity of such devices exploring other configurations and materials.

\section{REFERENCES}

[1] Y. Suzuki, H. Shimizu, M. Takeuchi, K. Nakamura, A.Yamada, Some studies on SAW resonators and multiple-mode filters, Proc. Of the IEEE Ultrasonics Symposium, pp. 297-302, 1976

[2] G. Scholl, C. Korden, E. Riha, C.C.W. Ruppel, U. Wolff, G. Riha, L. Reindl, R. Weigel, SAW-Based Radio Sensor Systems for Short-Range Applications, IEEE microwave magazine, pp. 68-76, December 2003

[3] Ballandras, S.; Lardat, R.; Penavaire, L.; Menage, P.; Chommeloux, L.; Huet, H.; Lestrat, C.; Pastureaud, T.; Haon, F.; Steichen, W.; Heider, G.; , "P1I-5 Micro-Machined, All Quartz Package, Passive Wireless SAW Pressure and Temperature Sensor," Ultrasonics Symposium, 2006. IEEE , vol., no., pp.1441-1444, 2-6 Oct. 2006

[4] K. Nakamura and H. Amano, Design of longitudinally coupled SAW resonator filters with Chebyshev, Proc. of the IEEE Int. Freq. Cont. Symp, pp.273-277, 1996

[5] C. Droit, G. Martin, S. Ballandras, J.-M Friedt A frequency modulated wireless interrogation system exploiting narrowband acoustic resonator for remote physical quantity measurement, Rev. Sci Instrum. Vol 81, Issue 5, 056103 (2010) 